\title{
A NOTE ON STABILITY OF TIME DELAY SYSTEMS
}

\author{
Frédéric Gouaisbaut * Dimitri Peaucelle * \\ * LAAS-CNRS \\ 7 avenue du Colonel Roche, 31077 Toulouse cedex 4 FRANCE \\ Email: \{fgouaisb, peaucelle\} @laas.fr
}

\begin{abstract}
This paper considers the robust stability of time delay systems by means of quadratic separation theory. Using this formalism both delay independent and delay dependent criteria are provided. In the nominal case, without uncertainties, our result is shown to be equivalent to other LMI-based results from the literature. Finally, an academic example is provided to show the effectiveness of this robust analysis approach.
\end{abstract}

Keywords: Quadratic separation, Linear time delay systems, Stability, Robustness.

\section{INTRODUCTION}

Time delay systems analysis and control have been intensively studied by the control community during the past decade due to both emerging adapted control theories and a significant need from applications in information technology (Gopalsamy, 1992; Richard, 2003). In this paper we aim at looking the fundamental problem of delay-dependent stability analysis. This is performed using techniques issued from robust control theory and published in (Peaucelle et al., 2005). An incidence of this approach is that it allows to deal naturally with robustness and time-delay issues simultaneously. Similar ideas can also be found in (Zhang et al., 2001) where, in input-output framework, scaled small-gain results are applied and compared to (Park, 1999; Li and De Souza, 1997; Moon et al., 2001).

The present work enters a long sequence of papers building Linear Matrix Inequality (LMI) results by applying Lyapunov-Krasovskii theory and finding appropriate bounding techniques. Pioneering results are due to (Li and De Souza, 1997; Park, 1999; Moon et al., 2001) then improved by (Han, 2004; Fridman, 2001) at the expense of introducing many new decision variables. Since then, new methods such as (Wu et al., 2004; Xu and Lam, 2005) give new LMIformulations with less decision variables and similar results on examples. A secondary contribution of the paper is to demonstrate theoretically that all these results are equivalent and can be reformulated with even less variables.

\section{Notations:}

$\mathrm{R}^{m \times n}$ is the set of $m$-by- $n$ real matrices. $\mathrm{C}^{+}$is the right hand side of the complex plane. $A^{T}$ is the transpose of the matrix $A . A^{\perp}$ is a full rank matrix whose columns span the null-space of $A .1$ and 0 are respectively the identity and the zero matrices of appropriate dimensions. For Hermitian matrices, $A>(\geq) B$ if and only if $A-B$ is positive (semi) definite. $\langle A\rangle$ stands for the symmetric matrix $\langle A\rangle=A+A^{T}$.

Problem statement:

Let the following uncertain time-delay system:

$$
\begin{cases}\dot{x}(t)=A(\Delta) x(t)+A_{d}(\Delta) x(t-h) & \forall t \geq 0 \\ x(t)=\phi(t) & \forall t \in[-h, 0]\end{cases}
$$

where $x(t) \in \mathrm{R}^{n}$ is the instantaneous state, $\phi$ is the initial condition and $A, A_{d} \in \mathrm{R}^{n \times n}$ are known constant matrices. The delay is assumed to be constant possibly known to be bounded in an interval including zero $h \in[0 \bar{h}]$, where $\bar{h}$ may be infinite if delay independent conditions are looked for.

The case of parametric LFT-modelled uncertainties is considered: the operator $\Delta$ is a constant matrix known to belong to a given set $\Delta$; the dependency of the 
model with respect to $\Delta$ is rational and writes as

$$
\begin{aligned}
& {\left[A(\Delta) A_{d}(\Delta)\right]} \\
& \quad=\left[A A_{d}\right]+B \Delta(1-D \Delta)^{-1}\left[C C_{d}\right]
\end{aligned}
$$

\section{QUADRATIC SEPARATION FOR ROBUST STABILITY OF TIME DELAY SYSTEMS}

The aim of this section is to derive robust stability analysis results by means of quadratic separation. First we recall the result of (Peaucelle et al., 2005) which is then applied for appropriate representations of system (1).

Consider two possibly non-square matrices $\mathcal{E}$ and $\mathcal{A}$ and an uncertain matrix $\nabla$ with appropriate dimensions that belongs to some set $\mathbb{W}$. We make no assumption on the uncertainty set $\mathbb{W}$. Although it is not needed in (Peaucelle et al., 2005), it is assumed here for simplicity that $\mathcal{E}$ is full column rank.

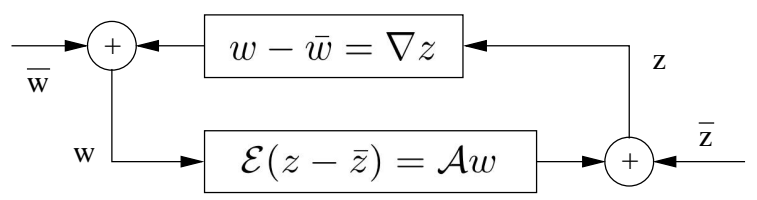

Figure 1. Feedback system

Theorem 1. The uncertain feedback system of Figure 1 is well-posed if and only if there exists a Hermitian matrix $\Theta=\Theta^{*}$ satisfying both conditions

$$
\begin{gathered}
{[\mathcal{E}-\mathcal{A}]^{\perp *} \Theta[\mathcal{E}-\mathcal{A}]^{\perp}>0} \\
{\left[1 \nabla^{*}\right] \Theta\left[\begin{array}{c}
1 \\
\nabla
\end{array}\right] \leq 0, \quad \forall \nabla \in \mathbb{W} .}
\end{gathered}
$$

If $\mathcal{E}$ and $\mathcal{A}$ are real matrices, the equivalence still holds with $\Theta$ restricted to be a real matrix.

Introducing the exogenous signals

$$
w_{\Delta}=\Delta z_{\Delta}, z_{\Delta}=C x+C_{d} x(t-h)+D w_{\Delta}
$$

Theorem 1 may be applied to the uncertain time delay system by rewriting system (1) with (2) as a feedback connected system of Figure 1 with $\mathcal{E}=1$,

$$
\mathcal{A}=\left[\begin{array}{ccc}
A & A_{d} & B \\
1 & 0 & 0 \\
C & C_{d} & D
\end{array}\right], \nabla=\left[\begin{array}{ccc}
s^{-1} 1 & 0 & 0 \\
0 & e^{-h s} 1 & 0 \\
0 & 0 & \Delta
\end{array}\right] .
$$

We aim at proving robust stability (i.e. no poles in the right hand side of the complex plane for all values of the delay and for all values of the uncertainty) which problem can be recast in the present framework as the well-posedness of the feedback connected system for all $s^{-1} \in \mathrm{C}^{+}$, all admissible values of $h \in[0 \bar{h}]$ and all admissible uncertainties $\Delta \in \Delta$. For an uncertainty set $\mathbb{W}$ defined in this way, a conservative choice of quadratic separator that fullfils (4) is

$$
\Theta=\left[\begin{array}{ccc|ccc}
0 & 0 & 0 & -P & 0 & 0 \\
0 & -Q & 0 & 0 & 0 & 0 \\
0 & 0 & T_{1} & 0 & 0 & T_{2} \\
\hline-P & 0 & 0 & 0 & 0 & 0 \\
0 & 0 & 0 & 0 & Q & 0 \\
0 & 0 & T_{2}^{*} & 0 & 0 & T_{3}
\end{array}\right]
$$

with $P>0, Q>0$ and

$$
\left[\begin{array}{ll}
1 & \Delta^{*}
\end{array}\right] \underbrace{\left[\begin{array}{ll}
T_{1} & T_{2} \\
T_{2}^{*} & T_{3}
\end{array}\right]}_{T}\left[\begin{array}{c}
1 \\
\Delta
\end{array}\right] \leq 0 \quad \forall \Delta \in \Delta .
$$

The choice of matrix $T$ must be done in accordance to the definition of the uncertainty set $\Delta$. Many such choices were proposed in the literature, see (Iwasaki and Hara, 1998) for a quite complete list. Here we suggest one for a particular case of uncertainties that fit with the example tested in the last section.

Assume $\Delta$ is a set of diagonal real valued matrices with bounded entries:

$$
\Delta=\left\{\Delta=\operatorname{diag}\left(\delta_{1}, \ldots \delta_{N}\right):\left|\delta_{i}\right| \leq \bar{\delta}_{i}\right\} .
$$

Define the set of all $2^{N}$ vertices of this convex set

$$
\Delta^{v}=\left\{\Delta=\operatorname{diag}\left( \pm \bar{\delta}_{1}, \cdots \pm \bar{\delta}_{N}\right)\right\} .
$$

Then a quadratic separator can be chosen such that (6) holds for the finite number of vertices in $\Delta^{v}$ and with $T_{3}$ having positive values on its diagonal:

$$
T_{3 i i} \geq 0,\left[\begin{array}{ll}
1 & \Delta^{*}
\end{array}\right] T\left[\begin{array}{c}
1 \\
\Delta
\end{array}\right] \leq 0 \quad \forall \Delta \in \Delta^{v} .
$$

The proof of the relevance of such separator and the fact that it leads to less conservative results than other known choices can be found in (Iwasaki and Hara, 1998).

With these definitions Theorem 1 applied to the robust stability of time delay systems gives the following Corollary.

Corollary 1. If there exist $P>0, Q>0$ and $T$ such that (8) and

$$
N_{1}^{T}\left[\begin{array}{ll}
0 & P \\
P & 0
\end{array}\right] N_{1}+N_{2}^{T}\left[\begin{array}{cc}
Q & 0 \\
0 & -Q
\end{array}\right] N_{2}<N_{3}^{T} T N_{3}
$$

where

$N_{1}=\left[\begin{array}{ccc}A & A_{d} & B \\ 1 & 0 & 0\end{array}\right] N_{2}=\left[\begin{array}{lll}1 & 0 & 0 \\ 0 & 1 & 0\end{array}\right] N_{3}=\left[\begin{array}{ccc}C & C_{d} & D \\ 0 & 0 & 1\end{array}\right]$ then system given by (1) and (2) with uncertainties as (7) is robustly stable whatever $h \geq 0$.

Proof: Note that for the given matrices

$$
[\mathcal{E}-\mathcal{A}]^{\perp}=\left[\begin{array}{c}
\mathcal{A} \\
1
\end{array}\right]
$$

The corollary is a direct application of Theorem 1 with separator (5). The result is delay independent since it does not depend on $\bar{h}$.

The result of this Corollary is not totally new. For example, in case of norm bounded uncertainties (and 
appropriate choices of separators) it can be found in (Gu et al., 2003, Chapter 6). The novelty is essentially to allowing many types of uncertainties in a unified framework.

We aim now at deriving delay dependent results with the same methodology. To do so note that the results were delay independent because the operator $e^{-h s}$, when $s^{-1} \in \mathrm{C}^{+}$, can only be characterized as norm bounded by 1 . To get delay dependent results it is therefore needed to have characteristics that depend on $\bar{h}$. This can be done noting that for all $s^{-1} \in \mathrm{C}^{+}$ and $h \in[0 \bar{h}]$ one has

$$
\left|s^{-1}\left(1-e^{-h s}\right)\right| \leq \bar{h}
$$

and this operator is such that

$$
V(s)=s^{-1}\left(1-e^{-h s}\right) \dot{X}(s)
$$

where $V(s)$ and $\dot{X}(s)$ are the Laplace transforms respectively of $v(t)=x(t)-x(t-h)$ and $\dot{x}(t)$. Introducing these new equation into the model leads to write the robust stability problem as a well-posedness problem of system in Figure 1 with

$$
\mathcal{E}=\left[\begin{array}{cccc}
1 & 0 & 0 & 0 \\
0 & 1 & 0 & 0 \\
0 & 0 & 1 & 0 \\
0 & 0 & 0 & 0 \\
1 & 0 & 0 & -1
\end{array}\right], \mathcal{A}=\left[\begin{array}{cccc}
A & A_{d} & B & 0 \\
1 & 0 & 0 & 0 \\
C & C_{d} & D & 0 \\
1 & -1 & 0 & 1 \\
0 & 0 & 0 & 0
\end{array}\right]
$$

and the augmented uncertain operator

$$
\nabla=\left[\begin{array}{cccc}
s^{-1} 1 & 0 & 0 & 0 \\
0 & e^{-h s} 1 & 0 & 0 \\
0 & 0 & \Delta & 0 \\
0 & 0 & 0 & s^{-1}\left(1-e^{-h s}\right) 1
\end{array}\right]
$$

Knowing the informations on every block of $\nabla$, a conservative choice of separator is

$$
\Theta=\left[\begin{array}{cccc|cccc}
0 & 0 & 0 & 0 & -P & 0 & 0 & 0 \\
0 & -Q & 0 & 0 & 0 & 0 & 0 & 0 \\
0 & 0 & T_{1} & 0 & 0 & 0 & T_{2} & 0 \\
0 & 0 & 0 & 0-\bar{h} R & 0 & 0 & 0 & 0 \\
\hline-P & 0 & 0 & 0 & 0 & 0 & 0 & 0 \\
0 & 0 & 0 & 0 & 0 & Q & 0 & 0 \\
0 & 0 & T_{2}^{*} & 0 & 0 & 0 & T_{3} & 0 \\
0 & 0 & 0 & 0 & 0 & 0 & 0 & \frac{1}{\bar{h}} R
\end{array}\right]
$$

with $P>0, Q>0, R \geq 0$ and $T$ satisfying (6). Noticing that

$$
[\mathcal{E}-\mathcal{A}]^{\perp}=\left[\begin{array}{ccc}
A & A_{d} & B \\
1 & 0 & 0 \\
C & C_{d} & D \\
A & A_{d} & B \\
\hline 1 & 0 & 0 \\
0 & 1 & 0 \\
0 & 0 & 1 \\
-1 & 1 & 0
\end{array}\right]
$$

Applying Theorem 1 gives the new delay dependent result:
Corollary 2. If there exist $P>0, Q>0, R \geq 0$ and $T$ such that (8) and

$$
\begin{aligned}
& N_{1}^{T}\left[\begin{array}{cc}
0 & P \\
P & 0
\end{array}\right] N_{1}+N_{2}^{T}\left[\begin{array}{cc}
Q & 0 \\
0 & -Q
\end{array}\right] N_{2} \\
& +N_{4}^{T}\left[\begin{array}{cc}
\bar{h} R & 0 \\
0 & -\frac{1}{\bar{h}} R
\end{array}\right] N_{4}<N_{3}^{T} T N_{3}
\end{aligned}
$$

where $N_{1,2,3}$ are those defined in Corollary 1 and

$$
N_{4}=\left[\begin{array}{ccc}
A & A_{d} & B \\
-1 & 1 & 0
\end{array}\right]
$$

then system given by (1) and (2) with uncertainties as (7) is robustly stable whatever $h \in[0 \bar{h}]$.

Corollary 1 can also be seen as a sub-case of Corollary 2 when taking $R=0$. It then renders the conditions independent on $\bar{h}$ and therefore gives conditions for possibly infinite delays.

Links with IQC methods:

To our knowledge quadratic separation (QS) framework as exposed presently was not utilized for the analysis of time delay systems while the most related framework of IQCs has proved to have applications for these (Megreski and Rantzer, 1997). To us the two approaches are complementary and most similar except that in IQC methods the Laplace operator is not considered with the same status as the other operators (delay, uncertainty). In the IQC framework it is then possible to get less conservative conditions at the expense of searching frequency dependent multipliers.

Related Lyapunov-Krasovskii functional:

Stability of the uncertain time delay system is tackled by means of quadratic separation in the previous subsection. We now prove that the results also correspond to conservative conditions for the existence of a Lyapunov-Krasovskii functional of the form:

$$
\begin{aligned}
V(t)= & x^{T}(t) P x(t)+\int_{t-h}^{t} x^{T}(\theta) Q x(\theta) d \theta \\
& +\int_{t-h}^{t} \int_{s}^{t} \dot{x}^{T}(\theta) R \dot{x}(\theta) d \theta d s
\end{aligned}
$$

Proposition 2. Under the LMI constraints $P>0$, $Q>0, R>0, T$ such that (6) and (12), the Lyapunov-Krasovskii functional (13) has its derivatives negative along the trajectories of system (1), therefore proving its asymptotic stability.

Proof: First note that if (12) is fulfilled then it also holds for any value of $h \in[0 \bar{h}]$ replacing $\bar{h}$. Define the vector

$$
\zeta^{T}(t)=\left(x^{T}(t) x^{T}(t-h) w_{\Delta}^{T}(t)\right)
$$

and note that

$$
N_{1} \zeta(t)=\left(\begin{array}{c}
\dot{x}(t) \\
x(t)
\end{array}\right), N_{2} \zeta(t)=\left(\begin{array}{c}
x(t) \\
x(t-h)
\end{array}\right)
$$




$$
N_{3} \zeta(t)=\left(\begin{array}{c}
z_{\Delta}(t) \\
\Delta z_{\Delta}(t)
\end{array}\right), N_{4} \zeta(t)=\left(\begin{array}{c}
\dot{x}(t) \\
v(t)
\end{array}\right)
$$

A congruence operation on (12) with $\bar{h}$ replaced by $h \in[0 \bar{h}]$ therefore gives

$$
\begin{array}{r}
2 x^{t}(t) P \dot{x}(t)+x^{T}(t) Q x(t)-x^{T}(t-h) Q x(t-h) \\
-z_{\Delta}^{T}(t)\left[\begin{array}{ll}
1 & \Delta^{T}
\end{array}\right] T\left[\begin{array}{c}
1 \\
\Delta
\end{array}\right] z_{\Delta} \\
+h \dot{x}^{T}(t) R \dot{x}(t)-\frac{1}{h} v^{T}(t) R v(t) \leq 0
\end{array}
$$

Due to (6) and Jensen's inequality (see ( $\mathrm{Gu}$ et al., 2003) and references therein) that states

$$
-\int_{t-h}^{t} \dot{x}^{T}(\theta) R \dot{x}(\theta) d \theta<-\frac{1}{h} z^{T}(t) R z(t)
$$

it implies that

$$
\begin{array}{r}
2 x^{t}(t) P \dot{x}(t)+x^{T}(t) Q x(t)-x^{T}(t-h) Q x(t-h) \\
+h \dot{x}^{T}(t) R \dot{x}(t)-\int_{t-h}^{t} \dot{x}^{T}(\theta) R \dot{x}(\theta) d \theta \leq 0
\end{array}
$$

which is exactly $\dot{V}(t) \leq 0$.

\section{COMPARISONS WITH OTHERS PAPERS}

Many papers in the literature are devoted to giving LMI conditions for delay-dependent analysis of stability of time delay systems. Some of these result have robust counterparts but with many different types of uncertain models. Therefore keeping in mind simplicity, the following comparison is done considering systems without uncertainties. In that case a corollary to Corollary 2 is that:

Corollary 3. Asymptotic stability of (1) with zero uncertainties $(\Delta=0)$ is proved for all delays $h$ such that $0 \leq h \leq \bar{h}$ if there exist three positive definite matrices $P, Q$ and $R$ such that:

$$
N_{4}^{T} \Lambda N_{4}<0
$$

where

$\Lambda=\left[\begin{array}{ccc}Q-R / \bar{h} & P & R / \bar{h} \\ P & \bar{h} R & 0 \\ R / \bar{h} & 0 & -Q-R / \bar{h}\end{array}\right], N_{4}=\left[\begin{array}{cc}1 & 0 \\ A & A_{d} \\ 0 & 1\end{array}\right]$

This result is now compared with respect to four previously published LMI based results. These four LMIformulas have been selected because: they have been produced recently almost in the same time; they prove to be better than earlier methods; all give quasi identical results on numerical examples. It is possible to prove that all these results including ours are in fact equivalent. Rather than proving totally this equivalence we prove that our result includes the previous ones. The converse is also true in some cases, it needs only to manipulate the formulas and add unnecessary additional variables.
The first considered result is from (Suplin et al., 2004). It follows several other results of the same authors labeled as "descriptor methods". We have selected this last formulation because the authors proved it to be equivalent to the previous ones.

Theorem 3. (Suplin et al., 2004, Theorem 4) The system (1) with zero uncertainty is asymptotically stable for any delay $h$ such that $0<h<\bar{h}$ if there exist $P_{1}>0, S>0 R>0 P_{i}, i=2, \ldots 4, Y_{1}, Y_{2}, Z_{1}, Z_{2}$ and $Z_{3}$ that satisfy the following LMIs:

$$
\begin{gathered}
\Psi=\left[\begin{array}{ccc}
R & Y_{1} & Y_{2} \\
Y_{1}^{T} & Z_{1} & Z_{2} \\
Y_{2}^{T} & Z_{2}^{T} & Z_{3}
\end{array}\right]>0 \\
\Xi+\left\langle\left[\begin{array}{c}
P_{2}^{T} \\
P_{3}^{T} \\
P_{4}^{T}
\end{array}\right]\left[\begin{array}{ccc}
A-1 & A_{d}
\end{array}\right]\right\rangle<0
\end{gathered}
$$

where

$\Xi=\left[\begin{array}{ccc}Y_{1}+Y_{1}^{T}+S+\bar{h} Z_{1} & P_{1}+Y_{2}+\bar{h} Z_{2} & -Y_{1}^{T} \\ \star & \bar{h}\left(R+Z_{3}\right) & -Y_{2}^{T} \\ \star & \star & -S\end{array}\right]$.

Proposition 4. If there exists a solution to the LMIs of Theorem 3 then conditions of Corollary 3 also hold with $P=P_{1}, Q=S$ and $R=R$.

Proof: First notice that for $P=P_{1}, Q=S, R=R$ :

$$
\Xi=\Lambda+\frac{1}{h}\left[\begin{array}{ccc}
1 & 0 & -1 \\
\bar{h} 1 & 0 & 0 \\
0 & \bar{h} 1 & 0
\end{array}\right]^{T} \Psi\left[\begin{array}{ccc}
1 & 0 & -1 \\
\bar{h} 1 & 0 & 0 \\
0 & \bar{h} 1 & 0
\end{array}\right]
$$

Since $\Psi>0$, inequalities (16) implies that

$$
\Lambda+\left\langle\left[\begin{array}{c}
P_{2}^{T} \\
P_{3}^{T} \\
P_{4}^{T}
\end{array}\right]\left[\begin{array}{ll}
A-1 & A_{d}
\end{array}\right]\right\rangle<0
$$

Notice that $\left[A-1 A_{d}\right]^{\perp}=N_{4}$. Applying Finsler lemma (Skelton et al., 1998) to this inequality concludes the proof.

A second result to be compared is given in (Han, 2004) and is also classified as a "descriptor method". For comparison purpose we reproduce it in the simplified case without the neutral and the distributed delay components.

Theorem 5. (Han, 2004, Corollary 1) The system (1) with zero uncertainty is asymptotically stable for a delay $\bar{h}$ if there exist $P_{1}>0, S>0, R>0, P_{2}$ and $P_{3}$ that satisfy the following LMIs:

$$
\left[\begin{array}{cccc}
\Xi_{11} & \Xi_{12} & P_{2}^{T} B_{2} & -\bar{h} P_{2}^{T} B_{1} \\
\star & \Xi_{22} & P_{3}^{T} B_{2} & -\bar{h} P_{3}^{T} B_{1} \\
\star & \star & -S & 0 \\
\star & \star & \star & -\bar{h} R
\end{array}\right]<0
$$

where $A_{d}=B_{1}+B_{2}$ and

$$
\begin{aligned}
& \Xi_{11}=P_{2}^{T}\left(A+B_{1}\right)+\left(A+B_{1}\right)^{T} P_{2}+S \\
& \Xi_{12}=P_{1}-P_{2}^{T}+\left(A+B_{1}\right)^{T} P_{3} \\
& \Xi_{22}=-P_{3}-P_{3}^{T}+h R
\end{aligned}
$$


Proposition 6. If there exists a solution to the LMIs of Theorem 5 then conditions of Corollary 3 also hold with $P=P_{1}, Q=S$ and $R=R$.

Proof: Recalling the $B_{1}+B_{2}=A_{d}$ one gets that:

$$
\begin{aligned}
& {\left[\begin{array}{cccc}
1 & 0 & 0 & 1 / \bar{h} \\
0 & 1 & 0 & 0 \\
0 & 0 & 1 & -1 / \bar{h}
\end{array}\right](17)\left[\begin{array}{cccc}
1 & 0 & 0 & 1 / \bar{h} \\
0 & 1 & 0 & 0 \\
0 & 0 & 1 & -1 / \bar{h}
\end{array}\right]^{T}} \\
& \quad=\Lambda+\left\langle\left[\begin{array}{c}
P_{2}^{T} \\
P_{3}^{T} \\
0
\end{array}\right]\left[\begin{array}{lll}
A & -1 & A_{d}
\end{array}\right]\right\rangle<0
\end{aligned}
$$

which concludes the proof for the same reasons as in the previous proof.

The third comparison is done with respect to results by $\mathrm{Xu}$ and Lam.

Theorem 7. (Xu and Lam, 2005, Theorem 1) The system (1) with zero uncertainty is asymptotically stable for any delay $h$ such that $0<h<\bar{h}$ if there exist matrices $P>0, Q>0, Z>0, Y$ and $W$ satisfying the LMI:

$$
\left[\begin{array}{cccc}
\Omega & P A_{d}-Y+W^{T} & -\bar{h} Y & \bar{h} A^{T} Z \\
\star & -Q-W-W^{T} & -\bar{h} W & \bar{h} A_{d}^{T} Z \\
\star & \star & -\bar{h} Z & 0 \\
\star & \star & \star & -\bar{h} Z
\end{array}\right]<0
$$

where $\Omega=A^{T} P+P A+Y+Y^{T}+Q$.

Proposition 8. If there exists a solution to the LMIs of Theorem 7 then conditions of Corollary 3 also hold with $P=P, Q=Q$ and $R=Z$.

Proof: Define the matrix

$$
\Phi_{1}=\left[\begin{array}{cccc}
A^{T} P+P A+Q & P A_{d} & 0 & \bar{h} A^{T} R \\
\star & -Q & 0 & \bar{h} A_{d}^{T} R \\
\star & \star & -\bar{h} R & 0 \\
\star & \star & \star & -\bar{h} R
\end{array}\right]
$$

then the LMI (18) with $R=Z$ writes also as:

$$
\Phi_{1}+\left\langle\Phi_{2}^{T}\left[\begin{array}{c}
Y \\
W
\end{array}\right] \Phi_{3}\right\rangle<0
$$

where the matrices $\Phi_{2}$ and $\Phi_{3}$ are such that

$$
\begin{gathered}
\Phi_{2}=\left[\begin{array}{llll}
1 & 0 & 0 & 0 \\
0 & 1 & 0 & 0
\end{array}\right] \\
\Phi_{3}^{T}=\left[\begin{array}{c}
1 \\
-1 \\
-\bar{h} 1 \\
0
\end{array}\right], \Phi_{3}^{\perp}=\left[\begin{array}{ccc}
1 & 0 & 0 \\
0 & 1 & 0 \\
1 / \bar{h} & -1 / \bar{h} & 0 \\
0 & 0 & 1
\end{array}\right]
\end{gathered}
$$

Applying Finsler lemma (Skelton et al., 1998) to this inequality implies: $\Phi_{3}^{\perp T} \Phi_{1} \Phi_{3}^{\perp}<0$, which gives when developed:

$$
\left[\begin{array}{ccc}
A^{T} P+P A+Q-R / \bar{h} & P A_{d}+R / \bar{h} & \bar{h} A^{T} R \\
\star & -Q-R / \bar{h} & \bar{h} A_{d}^{T} R \\
\star & \star & -\bar{h} R
\end{array}\right]<0
$$

It is exactly (14) if performing a Schur complement on the bottom right matrix $-h R$.
Finally, the last comparison is with respect to results by $\mathrm{Wu}$ et al. We have considered the simplified case of constant time delay which is the one adopted for comparisons. In that case the results write as:

Theorem 9. (Wu et al., 2004, Theorem 2) The system (1) with zero uncertainty is asymptotically stable for a delay $\bar{h}$ if there exist matrices $P>0, Q>0, Z>0$, $Y$ and $T$ such that the following LMIs are true:

$$
\underbrace{\left[\begin{array}{ccc}
\phi_{11} & \phi_{12} & \bar{h} A^{T} Z \\
\star & \phi_{22} & \bar{h} A_{d}^{T} Z \\
\star & \star & -\bar{h} Z
\end{array}\right]}_{\Upsilon_{1}}<0 \underbrace{\left[\begin{array}{ccc}
X_{11} & X_{12} & Y \\
\star & X_{22} & T \\
\star & \star & Z
\end{array}\right]}_{\Upsilon_{2}} \geq 0
$$

where

$$
\begin{aligned}
& \phi_{11}=A^{T} P+P A+Y+Y^{T}+Q+\bar{h} X_{11} \\
& \phi_{12}=P A_{d}-Y+T^{T}+\bar{h} X_{12} \\
& \phi_{22}=-T-T^{T}-Q+\bar{h} X_{22}
\end{aligned}
$$

Proposition 10. If there exists a solution to the LMIs of Theorem 9 then conditions of Corollary 3 also hold with $P=P, Q=Q$ and $R=Z$.

Proof: Simply note that under LMI conditions (20) one get that:

$$
\Upsilon_{1}<0 \leq \bar{h}\left[\begin{array}{ccc}
1 & 0 & 1 / \bar{h} \\
0 & 1 & -1 / \bar{h} \\
0 & 0 & 0
\end{array}\right] \Upsilon_{2}\left[\begin{array}{ccc}
1 & 0 & 0 \\
0 & 1 & 0 \\
1 / \bar{h} & -1 / \bar{h} & 0
\end{array}\right]
$$

Develop this inequality, it appears that all $X_{i j}, T$ and $Y$ matrices are eliminated and remains exactly condition (19) with $R=Z$.

\section{EXAMPLE}

Let consider the uncertain time delay system such that (1) and (2) with

$$
\begin{gathered}
A=\left[\begin{array}{cc}
-2 & 0 \\
0 & -1
\end{array}\right], A_{d}=\left[\begin{array}{cc}
-1 & 0 \\
-1 & -1
\end{array}\right] \\
B=\left[\begin{array}{ll}
1_{2} & 1_{2}
\end{array}\right],\left[C C_{d}\right]=1_{4}, D=0 \\
\Delta=\operatorname{diag}\left(\delta_{1}, \delta_{2}, \delta_{3}, \delta_{4}\right) \\
\left|\delta_{1}\right| \leq \bar{\delta}_{1}=1.6,\left|\delta_{2}\right| \leq \bar{\delta}_{2}=0.05 \\
\left|\delta_{3}\right| \leq \bar{\delta}_{3}=0.1,\left|\delta_{4}\right| \leq \bar{\delta}_{4}=0.3
\end{gathered}
$$

This example was extensively treated in many papers among which are those cited in the numerical comparisons of Table 1. Note that in some papers the example was modified into a system with a 2-by-2 norm bounded uncertainty. It corresponds to an optimistic modeling that may therefore not be compared to the real case. We have therefore re-tested ourselves all the methods. For those that need the uncertainty to be modeled as norm-bounded we took:

$$
\Delta\left[\begin{array}{ll}
C & C_{d}
\end{array}\right]=\tilde{\Delta}\left[\begin{array}{ll}
\tilde{C} & \tilde{C}_{d}
\end{array}\right]
$$

with $\tilde{\Delta}^{T} \tilde{\Delta} \leq 1$ and $\left[\tilde{C} \tilde{C}_{d}\right]=\operatorname{diag}\left(\bar{\delta}_{1}, \bar{\delta}_{2}, \bar{\delta}_{3}, \bar{\delta}_{4}\right)$. 
Table 1. Maximum allowable delay

\begin{tabular}{cllr}
\hline Method & Reference & $h_{\max }$ & nb.vars. \\
\hline 1 & (Li and De Souza, 1997) & 0.203 & 19 \\
2 & (Kim, 2001) & 0.2412 & 15 \\
3 & (Yue and Won, 2002) & 0.2412 & 15 \\
4 & (Xu and Lam, 2005) & 0.64 & 18 \\
5 & (Wu et al., 2004) & 0.64 & 29 \\
6 & (Moon et al., 2001) & 0.7059 & 18 \\
7 & (Fridman and Shaked, 2003) & 1 & 115 \\
8 & Corollary 2 & $\mathbf{1 . 0 1}$ & $\mathbf{1 9}$ \\
9 & (Suplin et al., 2004) & 1.61 & 333 \\
\hline
\end{tabular}

To comment the results of table 1 note that there are three sources of conservatism in the exposed methods:

(i) Choice of a delay-dependent Lyapunov functional and manipulations to get LMIs;

(ii) Choice of a modeling of uncertainties and manipulations to get LMIs;

(iii) Choice of a $\Delta$-dependent Lyapunov functional.

In terms of (i), methods 4, 5, 7, 8 and 9 are equivalent and are better that methods 1,2,3 and 6. This is proved in the previous section where we considered the case without uncertainties. In terms of (ii) method 8 is better than method 6 and they are better than methods $1,2,3,4$ and 5 since they exploit (partly for method 6 ) the diagonal structure of the uncertainty. Finally it must be pointed out that methods 7 and 9 are less conservative because of (ii) since they exploit the affine polytopic structure of the uncertain model and because of (iii) since they introduce parameter-dependent Lyapunov functionals. The fact that methods 7 and 8 give very close results is quite surprising when comparing the number of decision variables involved in the LMI constraints.

\section{CONCLUSION}

A new LMI formulation for delay dependent stability analysis is given. Compared to other methods it proves to be as conservative while taking advantage of fewer decision variables. The result is derived applying a new methodology for time-delay systems (namely the quadratic separation setup). The advantage of the new methodology being to naturally guide the choice of over-boundings on the uncertainty operator depending on its values and its structure.

\section{REFERENCES}

Fridman, E. (2001). New Lyapunov-Krasovskii functionals for stability of linear retarded and neutral type systems. Systems \& Control Letters 43(4), 309-319.

Fridman, E. and U. Shaked (2003). Delay-dependent stability and $h_{\infty}$ control: constant and time varying delay. International journal of control 76(1), 48-60.

Gopalsamy, K. (1992). Stability and Oscillations in Delay Differential Equations of Population Dynamics. Vol. 74 of Mathematics and Applications. Kluwer Acad.
Gu, K., K.L. Kharitonov and J. Chen (2003). Stability of Time-Delay Systems. Control Engineering Series. Birkhauser. Boston USA.

Han, Q.L. (2004). A descriptor system appraoch to robust stability of uncertain neutral systems with discrete dans distributed delays. Automatica 40, 1791-1796.

Iwasaki, T. and S. Hara (1998). Well-posedness of feedback systems: Insights into exact robustness analysis and approximate computations. IEEE Trans. Aut. Control 43(5), 619-630.

Kim, J.H. (2001). Delay and its time derivative dependent robust stability of time-delayed linear systems with uncertainties. IEEE Trans. Aut. Control 46, 789-792.

Li, X. and C.E. De Souza (1997). Delay-dependent robust stability and stabilization of uncertain linear delay systems : A linear matrix inequality approach. IEEE Trans. Aut. Control 42(8), 11441148.

Megreski, A. and A. Rantzer (1997). System analysis via integral quadratic constraints. IEEE Trans. Aut. Control 42(6), 819-830.

Moon, Y. S., P. Park, W. H. Kwon and Y. S. Lee (2001). Delay-dependent robust stabilization of uncertain state-delayed systems. Int. J. Control 74, 1447-1455.

Park, P. (1999). A delay-dependent stability criterion for systems with uncertain time-invariant delays. IEEE Trans. Aut. Control 44(4), 876-877.

Peaucelle, D., D. Henrion and D. Arzelier (2005). Quadratic separation for feedback connection of an uncertain matrix and an implicit linear transformation. In: 16th IFAC World Congress. Prague, Czech Republic.

Richard, J.P. (2003). Time delay systems: An overview of some recent advances and open problems. Automatica 39(10), 1667-1694.

Skelton, R.E., T. Iwazaki and K. Grigoriadis (1998). A unified Approach to Linear Control Design. Taylor and Francis series in Systems and Control.

Suplin, V., E. Fridman and U. Shaked (2004). A projection approach to $h_{\infty}$ control of time-delay systems. In: Proc. 43th IEEE CDC'04. Atlantis, Bahamas.

Wu, M., Y. He, J.H. She and G.P Liu (2004). Delay dependent criteria for robust stability of timevarying delay systems. Automatica 40, 14351439.

$\mathrm{Xu}, \mathrm{S}$. and J. Lam (2005). Improved delay-dependent stability criteria for time-delay systems. IEEE Trans. Aut. Control 50(3), 384-387.

Yue, D. and S. Won (2002). An improvement on 'delay and its time derivative dependent robust stability of time-delayed linear systems with uncertainties'. IEEE Trans. Aut. Control 47, 407-408.

Zhang, J., C.R. Knospe and P. Tsiotras (2001). Stability of time-delay systems: Equivalence between Lyapunov and scaled small-gain conditions. IEEE Trans. Aut. Control 46(3), 482-486. 